\title{
Contribution of the Putative Inner-Pore Region to the Gating of the Transient Receptor Potential Vanilloid Subtype 1 Channel (TRPV1)
}

\author{
Klara Susankova, ${ }^{1}$ Rudiger Ettrich, ${ }^{2}$ Ladislav Vyklicky, ${ }^{1}$ Jan Teisinger, ${ }^{1}$ and Viktorie Vlachova ${ }^{1}$ \\ ${ }^{1}$ Department of Cellular Neurophysiology, Institute of Physiology, Academy of Sciences of the Czech Republic, 14220 Prague 4, Czech Republic, and \\ ${ }^{2}$ Laboratory of High Performance Computing, Institute of Systems Biology and Ecology, Academy of Sciences of the Czech Republic and Institute of \\ Physical Biology, University of South Bohemia, 37333 Nove Hrady, Czech Republic
}

\begin{abstract}
The transient receptor potential vanilloid receptor-1 (TRPV1) is a sensory neuron-specific nonselective cation channel that is gated in response to various noxious stimuli: pungent vanilloids, low $\mathrm{pH}$, noxious heat, and depolarizing voltages. By its analogy to $\mathrm{K}^{+}$channels, the S6 inner helix domain of TRPV1 (Y666-G683) is a prime candidate to form the most constricted region of the permeation pathway and might therefore encompass an as-yet-unmapped gate of the channel. Using alanine-scanning mutagenesis, we identified 16 of 17 residues, that when mutated affected the functionality of the TRPV1 channel with respect to at least one stimulus modality. T670A was the only substitution producing the wild-type channel phenotype, whereas Y666A and N676A were nonfunctional but present at the plasma membrane. The periodicity of the functional effects of mutations within the TRPV1 inner pore region is consistent with an $\alpha$-helical structure in which T670 and A680 might play the roles of two bending "hinges."
\end{abstract}

Key words: capsaicin; vanilloid receptor; TRP channels; TRPV family; ion channel pores; structure-function relationship

\section{Introduction}

The gating of transduction ion channels in response to various external signals is a crucial mechanism underlying the function of sensory neurons. Among the ion channels that have been proposed to function as important signaling molecules involved in the perception of noxious chemical and thermal stimuli in primary afferent neurons, there is one specific channel with unique polymodal activation properties that have led to it be considered as a potential target for the treatment of pain: the transient receptor potential vanilloid type 1 (TRPV1) channel (Caterina et al., 1997). This nonselective cation channel can be activated by pungent vanilloid compounds such as capsaicin or resiniferatoxin, low $\mathrm{pH}(<6.5)$, noxious heat $\left(>43^{\circ} \mathrm{C}\right)$, and by voltage (Planells-Cases et al., 2005; Tominaga and Tominaga, 2005; Szallasi et al., 2006). These stimuli, when applied alone, produce only submaximal activation, whereas the ceiling for maximal response can only be reached by their synergistic interaction at the TRPV1 receptor (Tominaga et al., 1998; Vlachova et al., 2001). How does the vanilloid

\footnotetext{
Received Feb. 1, 2007; revised May 30, 2007; accepted May 30, 2007.

This work was supported by grants from the Czech Science Foundation (305/06/0319 and 303/07/0915), the Research Project Fund of the Academy of Sciences of the (zech Republic (AV0Z50110509 and AV0Z60870520), and the Ministry of Education, Youth, and Sports of the Czech Republic (1M0517, LC06010, MSM6007665808, and LC554). We thank Dr. D. Julius (University of California, San Francisco, CA) for providing the CDNA for rTRPV1 and Abdul Samad, Lina Zajats, and Thomas Stockner for assistance with computer modeling.

Correspondence should be addressed to Dr. Viktorie Vlachova, Institute of Physiology, Academy of Sciences of the Czech Republic, Videnska 1083, 14220 Prague 4, (zech Republic. E-mail: vlachova@biomed.cas.cz.

DOI:10.1523/JNEUROSCI.1956-07.2007

Copyright $\odot 2007$ Society for Neuroscience $\quad$ 0270-6474/07/277578-08\$15.00/0
}

TRPV1 channel transduce its specific stimuli (heat, capsaicin, voltage) into conformational changes to open the pore? And what are the structural determinants that control the gating processes? Despite intensive site-directed mutagenesis and biophysical studies on the mechanisms underlying TRPV1 activation, we are unable to account for all of the functional results obtained so far, mostly because we do not currently have enough information about the molecular principles of the gating of the TRPV1 channel.

Structurally, like other members of the TRP channel superfamily, TRPV1 channels are mainly homotetramers assembled with fourfold symmetry around a centrally located aqueous pore (Owsianik et al., 2006). Each subunit contains six transmembrane-spanning domains (S1-S6) with a porelining $\mathrm{P}$ region linking the S5 and S6 domains. The regions within the $\mathbf{S 6}$ segment are most strongly conserved among the TRP channel subfamilies; in particular, a sequence of five amino acid residues, NMLIA, is completely conserved across TRP-related channels, differing only in a leucine-to-tyrosine substitution in voltage-gated sodium channels (Kuzhikandathil et al., 2001). Although the atomic-resolution structure is not yet available for any of the TRP channels, it is generally assumed that their S5-P-S6 regions are potentially analogous to the molecular architecture of the two membrane-spanning $\alpha$ helices (TM1 and TM2) of a bacterial $\mathrm{K}^{+}$channel KcsA (Dodier et al., 2004; Ferrer-Montiel et al., 2004; Voets et al., 2004a; Nilius et al., 2005a; Yeh et al., 2005). The recently solved crystal structures of the potassium channels in their closed (Doyle et al., 1998) and open (Jiang et al., 2002) con- 
formations provide a general model of the pore region structure and suggest common principles for the gating of many other P-loop-containing channels, and thus quite possibly also for the gating of channels from the TRP family.

Here, we used alanine-scanning mutagenesis of the putative inner pore region of the rat vanilloid TRPV1 receptor, namely the amino acid sequence Y666-G683 that contains the most conserved region of the TRP protein family, with the aim of identifying candidate residues that could play a key role in the gating of the channel. We set out to clarify whether there is a structural analogy between the gating mechanism of potassium channels and the vanilloid receptor by molecular modeling of the TRPV1 (S5-P-S6) ${ }_{4}$ pore module and by taking the atomic structure of the Streptomyces lividans KcsA channel as a scaffold.

\section{Materials and Methods}

Cell culture and transfection. HEK293T cells were cultured in OPTI-MEM I medium (Invitrogen, Gaithersburg, MD) supplemented with 5\% FBS. HEK293T cells were transiently cotransfected with $400 \mathrm{ng}$ of cDNA plasmid encoding wild-type or mutant rat TRPV1 (wild type in the pcDNA3 vector kindly provided by Dr. D. Julius, University of California, San Francisco, CA) and with 400 ng of green fluorescent protein (GFP) plasmid (TaKaRa, Shiga, Japan) per $1.6 \mathrm{~mm}$ dish using either the Lipofectamine 2000 (Invitrogen, San Diego, CA) or Magnet-assisted Transfection (IBA, Louvain-la-Neuve, Belgium) method. Cells were used 24-48 h after transfection. For each experimental group, five to eight GFP-positive cells per coverslip were studied from at least two independent transfections.

Site-directed mutagenesis. Alanine mutants were generated by PCRmediated mutagenesis using the QuikChange XL Site Directed Mutagenesis kit (Stratagene, La Jolla, CA). All site-directed mutated constructs were confirmed by DNA sequencing using an automated sequencer (ABI PRISM 3100; Applied Biosystems, Foster City, CA).

Surface biotinylation assay. Biotinylation and purification of cellsurface proteins was performed using the Cell Surface Protein Isolation kit protocol (Pierce Biotechnology, Rockford, IL). After $48 \mathrm{~h}$ of transfection with wild-type or mutant TRPV1, HEK293T cells (grown in T25 tissue culture flasks) were rinsed twice in PBS and biotinylated with membrane-impermeable EZ-Link Sulfo-NHS-SS-biotin in PBS for 30 $\min$ at $4^{\circ} \mathrm{C}$. The biotinylation reaction was quenched, and the cells were washed and lysed in lysis buffer containing a HALT protease inhibitor mixture (Pierce Biotechnology). Protein concentration was determined using the Coomassie Plus Bradford Assay kit (Pierce Biotechnology). Samples of equal proteins were separated by SDS-PAGE and transferred onto nitrocellulose membrane (Amersham Biosciences, Freiburg, Germany). Immunoblots were blocked with $5 \%$ milk, $0.5 \%$ BSA, and $0.1 \%$ Tween 20 in Tris-buffered saline, $\mathrm{pH}$ 7.4, and probed with a TRPV1 $\mathrm{N}$-terminal antibody $(1: 2000)$ overnight at $4^{\circ} \mathrm{C}$. After washing with TTBS (20 mu Tris, $150 \mathrm{~mm} \mathrm{NaCl}$, and $0.1 \%$ Tween 20, pH 7.4), membranes were incubated with a horseradish peroxidase-conjugated donkey antirabbit secondary antibody (1:2500, $1 \mathrm{~h}$ at room temperature; Millipore, Billerica, MA). For detection, we used SuperSignal West Pico Chemiluminiscent Substrate (Pierce Biotechnology). Immunoblots were digitized using the LAS-1000 camera system (Fujifilm Corporation, Tokyo, Japan) and processed using the public-domain software ImageJ (version 1.37, http://rsb.info.nih.gov/ij/; National Institutes of Health, Bethesda, $\mathrm{MD})$.

Electrophysiology. Whole-cell membrane currents were recorded by using an Axopatch-1D amplifier and pClamp9 software (Molecular Devices, Sunnyvale, CA). A system for fast superfusion of the cultured cells was used for drug application and thermal stimulation (Dittert et al., 2006). The extracellular control solution contained (in mM) $160 \mathrm{NaCl}$, $2.5 \mathrm{KCl}, 1 \mathrm{CaCl}_{2}, 2 \mathrm{MgCl}_{2}, 10 \mathrm{HEPES}$, and 10 glucose, adjusted to $\mathrm{pH} 7.3$ and $315 \mathrm{mOsm}$. The pipette solution contained (in mM) $125 \mathrm{Cs}-$ gluconate, $15 \mathrm{CsCl} 5$ EGTA, $10 \mathrm{HEPES}, 0.5 \mathrm{CaCl}_{2}$, and $2 \mathrm{MgATP}(\mathrm{pH}$ 7.3, $283 \mathrm{mOsm}$ ). All chemicals were purchased from Sigma-Aldrich (St. Louis, MO). Capsaicin solution was prepared from a $0.1 \mathrm{~m}$ stock solution in ethanol and stored at $-20^{\circ} \mathrm{C}$. The final concentration of ethanol was $<0.01 \%$.

Statistical analysis. Voltage-dependent gating parameters were estimated from steady-state current-voltage $(I-V)$ relationships obtained at the end of $60 \mathrm{~ms}$ voltage steps from -140 to $+140 \mathrm{mV}$ by fitting them to the following Boltzmann equation: $I=g \times\left(V-E_{\mathrm{rev}}\right) /(1+\exp$ $\left.\left(-z F\left(V-V_{1 / 2}\right) / R T\right)\right)$, where $z$ is the equivalent charge, $V_{1 / 2}$ is the halfactivation voltage, $g$ is the whole-cell conductance, $E_{\text {rev }}$ is the reversal potential, and $F, R$, and $T$ have their usual thermodynamic meaning. Boltzmann fits were achieved with pClamp9 software (Molecular Devices). Data were routinely discarded if the leak exceeded $5 \%$ of the maximum amplitude, but for all the data in the current-voltage relationship experiments, the leak currents were $<1 \%$ of the maximum current, and therefore no leak subtraction was used. The heat-evoked whole-cell currents sampled during the rising phase of the temperature ramp were pooled for every $0.25^{\circ} \mathrm{C}$. The temperature coefficients $Q_{10}$ and the thermal thresholds were determined as described previously (Vlachova et al., 2003). $Q_{10}$ values were determined by using the following formula: $Q_{10}=$ $\exp \left(\Delta T \times E_{\mathrm{a}} /(R T 1 T 2)\right)$, where $R$ is the gas constant, $\Delta T=10$ Kelvin $(\mathrm{K})$, and $E_{\mathrm{a}}$ is an apparent activation energy estimated from the slope of Arrhenius plot between absolute temperatures $T 1$ and $T 2$. The thermal thresholds for wild-type and TRPV1 mutant activation were measured from the temperatures at which the inward currents plotted on a logarithmic scale declined significantly from a straight line. For this purpose, an adapted piecewise linear regression was used, via SigmaPlot 9 software (Systat Software, San Jose, CA). All data are presented as mean \pm SEM. Statistical significance was determined by ANOVA, followed by a post hoc Dunnett's test to determine levels of significance between wild-type and mutant receptors, if not stated otherwise, and differences were considered significant at $p<0.05$. Paired Student's $t$ tests and Pearson productmoment correlation analysis were used to assess the significance of statistical differences and the correlation coefficient $r$ between the pairs of current responses to 1 and $30 \mu \mathrm{M}$ capsaicin measured in wild-type and mutants at $47^{\circ} \mathrm{C}$.

Molecular modeling. The three-dimensional model of the S5-P-S6 pore module of rat TRPV1, including all nonhydrogen atoms, was built and examined by the Modeler version 6 package (Sali and Blundell, 1993). The tertiary structure model was checked with Procheck (Laskowski et al., 1993). The final proposed model was fitted onto the tetrameric structure of the KcsA channel to create the tetramer and energetically minimized by steepest descent minimization. The tetramer was then embedded into a preequilibrated palmitoyl oleoyl phosphatidylcholine (POPC) bilayer made from 512 lipid molecules following the general procedure for embedding a protein into the bilayer using the make_hole version of GROMACS 3.1 and the gmx forcefield adapted for POPC lipids. The production dynamics simulations, without any restraints, were $20 \mathrm{~ns}$ long and were run with GROMACS 3.2 (Van Der Spoel et al., 2005) using the gmx force field, with a 2 fs time step. SETTLE (for water) and LINCS (non-water) algorithms were used to constrain covalent bond lengths, and long-range electrostatic interactions were computed using the Particle-Mesh Ewald method. The temperature was kept at $300 \mathrm{~K}$ by separately coupling the protein and solvent to an external temperature bath $(\tau=0.1 \mathrm{ps})$ (Berendsen et al., 1984). The pressure was kept constant at 1 bar by weak coupling ( $\tau=1.0 \mathrm{ps}$ ) to a pressure bath using semiisotropic mode. The protein proved to be stable during simulation. The molecular dynamics (MD) run produced slightly different conformations of the four monomers that, in all cases, showed a bending of the lower end of the transmembrane helix. The monomer with the highest degree of preserved secondary structure during the run was used for the creation of a new tetramer that was again used for repeating the procedure of embedding the structure into the bilayer with the following $20 \mathrm{~ns}$ MD simulation.

\section{Results}

Alanine scanning in the putative inner-pore helix of TRPV1 reveals two nonfunctional mutants

Consecutive residues (Y666-G683) in the putative inner-pore region of the cloned rat TRPV1 channel were substituted, one at a time, by alanine, and the mutant channels were expressed in 

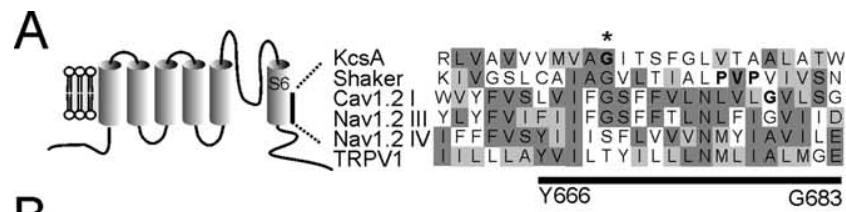

B

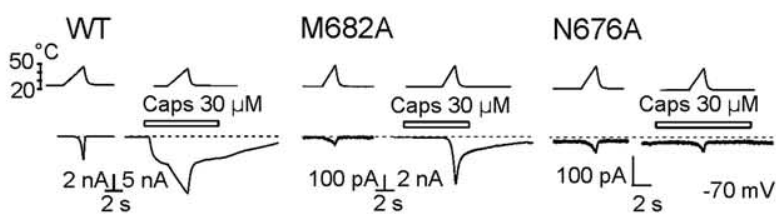

C
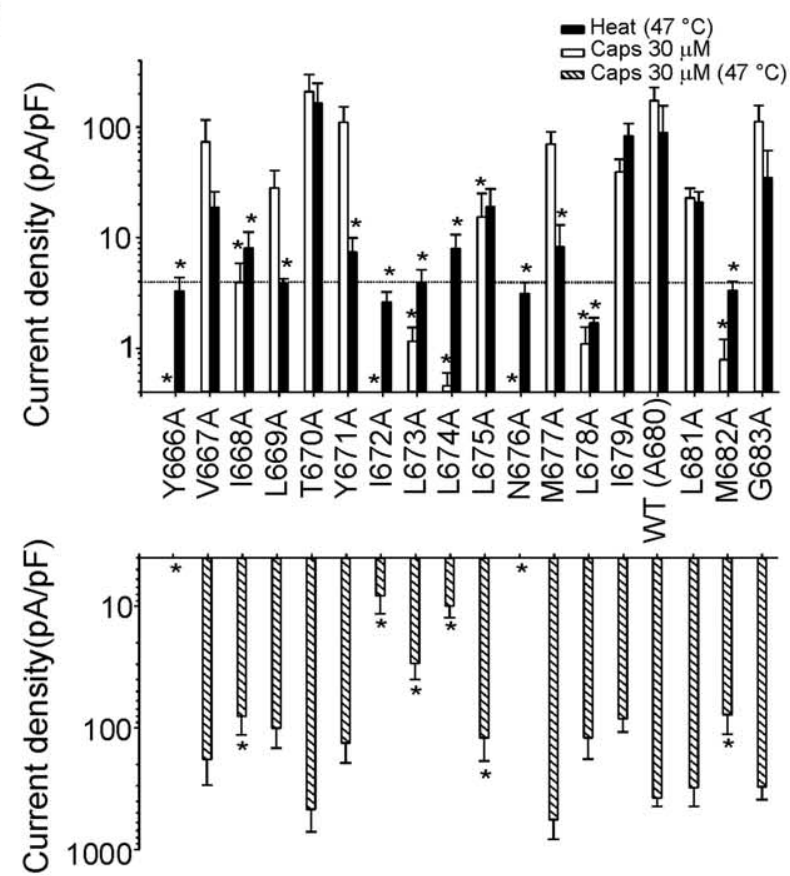

D
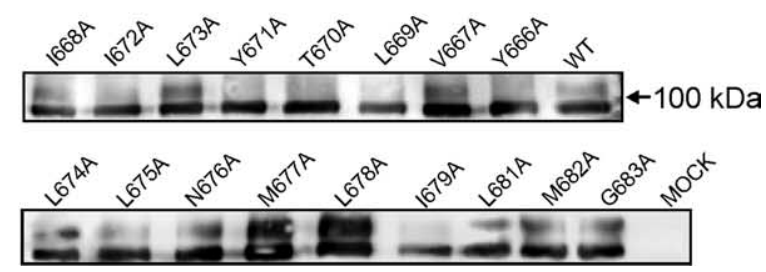

Figure 1. Alanine-scanning mutagenesis of the inner-pore region (Y666-G683) of the TRPV1 channel. $A$, Putative secondary structure of the TRPV1 channel subunit. Comparison of the inner-pore region of rat TRPV1 (GenBank accession number AAC53398) with that of KcsA (P0A334), Shaker (CAA29917), Ca 1.2 I (P15381), and Na 1.2 III and IV (NP_036779) proteins is shown. Protein sequences were aligned using MUSCLE multiple alignment software (http:// phylogenomics.berkeley.edu/cgi-bin/muscle/input_muscle.py) with Blosum62 score colors. In KcsA and Shaker, sequences were manually adjusted to specifically align conserved mid-glycine residues. Conserved amino acid residues proposed to serve as gating hinges in some of the channels are indicated with an asterisk and with bold type. $\boldsymbol{B}$, Representative whole-cell currents evoked by heat stimuli with or without $30 \mu \mathrm{m}$ capsaicin at $-70 \mathrm{mV}$ in wild-type TRPV1 and in M682A and N676A mutants. Bath temperature is plotted above. Open bars indicate duration of drug application. Dashed lines indicate zero current level. C, Summary of alaninescanning mutagenesis results. Bar graphs show average whole-cell current densities evoked at $25^{\circ} \mathrm{C}$ by $30 \mu \mathrm{m}$ capsaicin and by heat $\left(47^{\circ} \mathrm{C}\right.$ ) (top graph) and whole-cell current densities induced by $30 \mu \mathrm{m}$ capsaicin at $47^{\circ} \mathrm{C}$ for each mutant (bottom graph). The dotted line indicates 4 $\mathrm{pA} / \mathrm{pF}$, which roughly corresponds to the estimated amplitude of thermally induced nonspecific currents at $47^{\circ}$. Each bar is the mean \pm SEM of at least four independent cells. Statistical significance is indicated $\left({ }^{*} p<0.05\right)$. $\boldsymbol{D}$, Western blot of surface-expressed wild-type and alanine mutants of TRPV1 using an anti N-terminal TRPV1 antibody after surface biotinylation of cells transfected with each receptor or mock transfection control (see Materials and Methods for details).
HEK293T cells. The activation properties of the mutants were determined by recording whole-cell currents with a stimulation protocol consisting of depolarizing voltage steps recorded in standard extracellular solution at $25^{\circ} \mathrm{C}$ (from -140 to $+140 \mathrm{mV}$; increment, $+20 \mathrm{mV}$ ), brief exposure to capsaicin (1 and $30 \mu \mathrm{M}$ for $8 \mathrm{~s})$, and a $25-48^{\circ} \mathrm{C}$ heat ramp $\left(10^{\circ} \mathrm{C} / \mathrm{s}\right)$ applied before, during, and after capsaicin applications. Particularly in weakly responding mutants, the effects of extracellular protons ( $\mathrm{pH} 5)$ were examined using a desensitization protocol, in which the fast inactivating currents attributable to the endogenous protongated conductance mediated by native ASIC channels in HEK293T cells were inhibited by a 1 min application of $\mathrm{pH} 6.8$ (Gunthorpe et al., 2001). Capsaicin and heat sensitivities were also tested in calcium-free extracellular solution, and the time of exposure to capsaicin was prolonged in those channel mutants that had altered kinetics. The averaged results are summarized in Figure 1C.

At a holding potential of $-70 \mathrm{mV}$, the mean peak heatinduced current densities measured at $47^{\circ} \mathrm{C}$ were significantly reduced in 11 of 17 mutants compared with wild-type TRPV1 $(p<0.001$, one-way ANOVA with Dunnett's post hoc test). There were nine mutant channels that showed significantly reduced peak current densities when exposed to a supramaximal concentration of capsaicin $(30 \mu \mathrm{M})$ at room temperature $\left(25^{\circ} \mathrm{C}\right)$. Three mutant channels (Y666A, I672A, N676A) showed no capsaicin-evoked activity at all. These mutants were also $\mathrm{pH}$ insensitive, they were not activated in the absence of extracellular $\mathrm{Ca}^{2+}$, and they gave very small and nonspecific heat-induced membrane currents (without a marked activation threshold), usually not exceeding $-150 \mathrm{pA}$ at $48^{\circ} \mathrm{C}$.

Capsaicin and heat stimuli $\left(>43^{\circ} \mathrm{C}\right)$ applied together produce a strong synergistic activation of the wild-type TRPV1 channel. By using a heat stimulus $\left(47^{\circ} \mathrm{C}\right)$ in combination with a supramaximal concentration of capsaicin $(30 \mu \mathrm{M})$, we hoped we might also unmask functionality in some of the poorly sensitive mutants. Indeed, an efficient potentiation was, among other constructs, also found in the I672A, L673A, L674A, L678A, and M682A mutants, which were functionally silent or only barely responded to capsaicin at base temperature (Fig. 1C). Compared with wild-type TRPV1, the current densities of the capsaicininduced responses measured at $47^{\circ} \mathrm{C}$ were significantly reduced in eight mutants: Y666A, I668A, I672A-N676A, and M682A ( $p<$ 0.001 , one-way ANOVA with Dunnett's post hoc test). All of them also showed a significant reduction in the capsaicin-evoked current densities at $25^{\circ} \mathrm{C}$.

For these latter eight mutants, the decreases in capsaicin- and heat-induced current densities described above could arise from alterations in channel functionality but also from alterations in plasma membrane expression. To determine whether differences in current densities reflected changes in surface expression, we used cell-surface biotinylation and streptavidin precipitation to examine the surface expression of wild-type and mutant receptors. As shown in Figure $1 D$, Western blots of all of the mutants examined confirmed that the proteins were processed and targeted to the membrane to a similar extent as that of wild-type TRPV1.

Together, these results suggest that (1) there are two residues within the inner-pore region of rat TRPV1 that are critical for channel functionality: the aromatic residue in position Y666 and the highly conserved polar residue N676; and (2) among the mutants tested, L669A, Y671A, M677A, and L678A are significantly less sensitive to heat; the latter mutant also displayed a reduced sensitivity to capsaicin at $25^{\circ} \mathrm{C}$. 

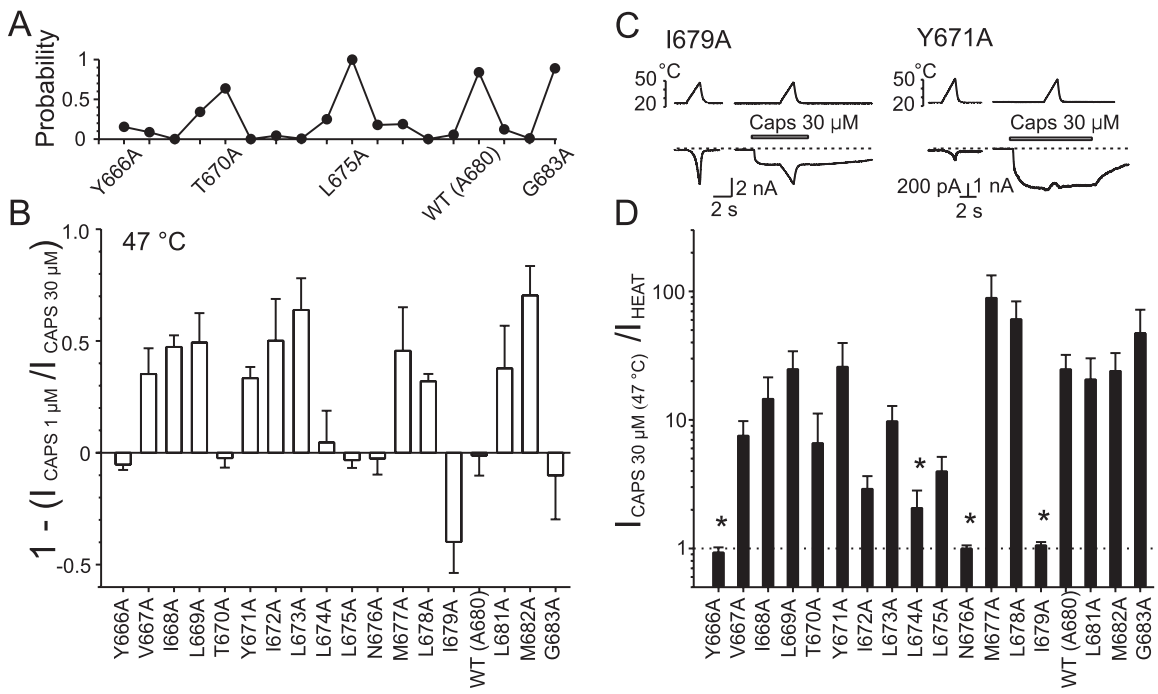

Figure 2. Changes in capsaicin sensitivity introduced by alanine mutations in putative inner-pore region of TRPV1.A, Probabilities that maximal responses to $1 \mu \mathrm{m}$ capsaicin measured at $47^{\circ} \mathrm{C}$ are equal to those induced by $30 \mu \mathrm{m}$ capsaicin at $47^{\circ} \mathrm{C}$. Each point represents the $p$ value obtained from paired Student's $t$ test. $\boldsymbol{B}$, Summary of alanine-scanning mutagenesis results comparing relative sensitivity to capsaicin. The bar graph shows mean whole-cell currents evoked by $1 \mu \mathrm{m}$ capsaicin at $47^{\circ} \mathrm{C}$ normalized to responses to $30 \mu \mathrm{m}$ capsaicin measured at $47^{\circ} \mathrm{C}$ for each construct. Each bar is the mean $\pm \mathrm{SEM}$ of at least four independent cells. C, Representative whole-cell currents evoked by heat stimuli applied with or without $30 \mu \mathrm{m}$ capsaicin at $-70 \mathrm{mV}$, recorded in 1679A and Y671A mutants. D, The bar graph shows average ratios of capsaicin- to heat-induced whole-cell currents measured both at $47^{\circ} \mathrm{C}$ in HEK293T cells expressing wild-type or alanine mutants of TRPV1. Statistical significance is indicated $\left({ }^{*} p<0.05\right)$. The dashed line indicates no potentiation.

\section{Relative sensitivity of alanine mutants to capsaicin}

Replacing tyrosine Y671 with alanine dramatically lowers the half-maximal activating capsaicin concentration $\mathrm{EC}_{50}$ by almost 1 order of magnitude (from 240 to $40 \mathrm{~nm}$ ) (Mohapatra et al., 2003). Specifically, in this mutant, we found that responses to capsaicin were not potentiated by heat. In contrast to the other mutants and the wild-type channel, the capsaicin-evoked responses were consistently inhibited by heating above $30^{\circ} \mathrm{C}$ (Fig. $2 C$ ). We reasoned that the loss of potentiation in Y671A might be attributable to changes in agonist sensitivity. Indeed, when the channels were desensitized by repeated applications of capsaicin ( $1 \mu \mathrm{M}$ and/or $30 \mu \mathrm{M}$ ), the heat-induced potentiation reappeared. To also explore the capsaicin responsiveness in other mutants, we compared their relative sensitivities at two different concentrations of capsaicin $(1$ and $30 \mu \mathrm{M})$. To overcome the potential effects of desensitization and to compare the magnitude of capsaicin-evoked currents in different mutants regardless of variations in expression levels, the inward currents induced by $1 \mu \mathrm{M}$ capsaicin were measured at $47^{\circ} \mathrm{C}$ and related to the responses induced by $30 \mu \mathrm{M}$ capsaicin, also measured at $47^{\circ} \mathrm{C}$ for each cell (Fig. 2A,B). Plotting the resulting $p$ values generated from the Student's paired $t$ test for significant differences between the pairs of 1 and $30 \mu \mathrm{M}$ capsaicin-evoked currents at $47^{\circ} \mathrm{C}$ against the appropriate mutation number (as shown in Fig. $2 \mathrm{~A}$ ), we found an alternating pattern with high values in T670A, L675A, G683A, and wild-type channels $(p>0.6)$ and the lowest values $(p<$ 0.05) in mutations I668A, Y671A-L673A, L678A, and M682A. These data are pooled and summarized in Figure $2 B$.

To further assess the maximal capsaicin-induced responses in the mutants, we compared the inward currents measured at $47^{\circ} \mathrm{C}$ in the absence and presence of a high concentration of capsaicin. Except for Y666A, N676A, and I679A, the inward currents were significantly $(p<0.01 ; n=4-11)$ greater in the presence of 30 $\mu \mathrm{M}$ capsaicin than in its absence (Fig. $2 D$ ). The lack of mutual potentiation between the two stimuli in the former two mutants was caused by their insensitivity to capsaicin. Notably, the mutation of isoleucine residue 679 retained normal sensitivities to both stimuli, although it completely removed their mutual potentiation at $47^{\circ} \mathrm{C}$. It should be noted that, in several residues, the degree of the capsaicin-induced increase in the amplitude of the inward current at $47^{\circ} \mathrm{C}$ was clearly reduced, although these changes did not always achieve statistical significance (e.g., V667A, T670A, I672A, L673A, and L675A).

Two interesting features arise from these data. (1) The periodicity observed in the relationships between the maximal activation capacities obtained for 1 and 30 $\mu \mathrm{M}$ capsaicin at $47^{\circ} \mathrm{C}$ supports the hypothesis that there exists a structural explanation for the gating of the TRPV1 channel by chemical stimuli. More speculatively, the pattern of sensitivity to capsaicin (every three to four substitutions) is consistent with an $\alpha$-helical structure contributing to capsaicin-induced channel gating. (2) Changes induced by individual mutations in the $1 \mu \mathrm{M}$ versus $30 \mu \mathrm{M}$ capsaicininduced current relationships measured at $47^{\circ} \mathrm{C}$ might reflect the changes either in capsaicin sensitivity or in the allosteric linkage between capsaicin and heat sensor movements and channel opening. Specifically, mutation I679A appears to cause a decrease in the synergistic interaction of capsaicin and heat with the TRPV1 receptor.

\section{Heat-evoked membrane currents in mutant channels}

As shown in Figure 1C, the mutations in most cases blunted the heat-induced current responses. To obtain a more complete picture of how the individual mutations affect the heat sensitivity of the TRPV1 channel and to further characterize the specificity of their temperature-induced responses, we quantified the temperature threshold for activation and the temperature coefficient $\left(Q_{10}\right)$ from the Arrhenius plots of individual current-temperature relationships. This approach allowed us to compare the heat sensitivities of different mutants, regardless of the wide cell-tocell variation in the size of the heat-evoked currents and the receptor expression levels. At a membrane potential of $-70 \mathrm{mV}$, the mean threshold for the temperature-induced membrane currents measured in the wild-type channel was $42.3 \pm 0.9^{\circ} \mathrm{C}$, and the median $Q_{10}$ was $37.4(n=10)$, which is in good agreement with values reported previously (Vlachova et al., 2003). Among the mutants tested, the most frequently observed phenotype was a leftward shift in the temperature threshold (Fig. $3 A, B$ ). Seven mutations had thermal thresholds no different from wild-type TRPV1. In the wild-type TRPV1 channel, $Q_{10}$ values calculated for the temperature range $43-48^{\circ} \mathrm{C}$ vary markedly, ranging from units to several tenths (Vlachova et al., 2003). To achieve statistical significance in some of the mutants would require an unrealistically large number of cells to be tested for each group. Qualitatively, however, it was evident that, except for the T670A, L681A, and G683A, the mutations strongly reduced $Q_{10}$ in all mutants tested.

To further compare the temperature sensitivities regardless of 
the size of current magnitude, the first temperature-evoked response of each cell was normalized at $45^{\circ} \mathrm{C}$ and averaged for each mutant. The resulting average temperature-dependent activation profile shown in Figure $4 A$ gives an immediate impression that the five peaks separated by four troughs at residues I672-L674, N676, L678, and M682 might reflect changes introduced by single mutations in an $\alpha$-helical structure, which most likely represents the inner-pore region of the TRPV1 channel.

\section{Effects of mutations on}

\section{voltage-dependent activation}

At room temperature and normal $\mathrm{pH}$ (7.3), rat cloned TRPV1 can be activated by depolarizing voltages in the absence of any agonists (Vlachova et al., 2003), and this mode of activation seems to be linked to conformational changes associated with temperature-dependent gating of the TRPV1 channel (Brauchi et al., 2004; Voets et al., 2004b; Nilius et al., 2005b). To compare the effects of mutations on voltage-dependent activation, we used a voltage step protocol from -140 to $+140 \mathrm{mV}$ (increment, +20 $\mathrm{mV})$ at $25^{\circ} \mathrm{C}$ and normalized the resulting current-voltage relationships at +60 $\mathrm{mV}$ for each cell (Fig. 3C). In the wildtype channel, the midpoint voltage of activation $\left(V_{1 / 2}\right)$ estimated by Boltzmann fit was $126 \pm 3.5 \mathrm{mV}(n=7)$ at $25^{\circ} \mathrm{C}$, which was in general agreement with previously published data (Voets et al., 2004b). Except for T670A, Y671A, and M677A, the alanine mutations led to a rightward shift of the activation curve and a concomitant decrease in the equivalent charge $z$ (Fig. 3D), reflecting a decrease in channel activity at more negative holding potentials. Even for voltage protocols up to $+140 \mathrm{mV}$, the open probability for some of the TRPV1 mutants was apparently below 0.5 , so that values for $V_{1 / 2}$ could not be reliably obtained from a Boltzmann fit in some cells (specifically in L681A and L678A). Despite this limitation, these data indicate that most of the alanine mutations modulate the gating of TRPV 1 by shifting the voltage dependence toward more positive membrane potentials.

As shown in Figure 4, $B$ and $C$, the average profile of the voltage-dependent activation for all mutants was not entirely analogous to their temperature-dependent activation profile. At the maximum voltage stimulation, the local maxima around L669, M677-L678, and L681 were separated by three troughs around residues I672N676, I679, and M682. These results support the view that temperature- and voltage-dependent mechanisms underlying the
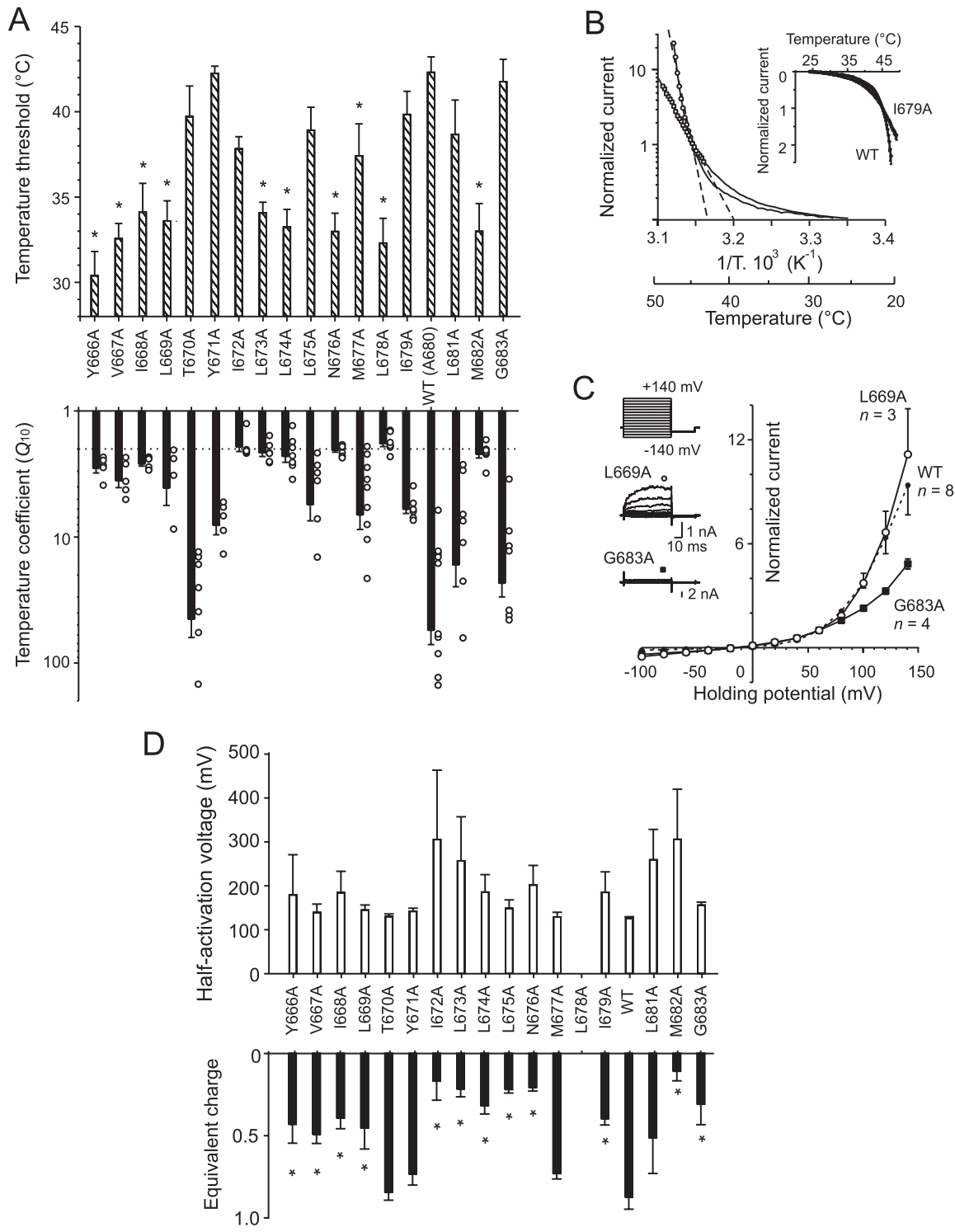

Figure 3. Changes in heat sensitivity produced by alanine mutations in the putative inner-pore region of TRPV1. $\boldsymbol{A}$, Bar graphs show effects of alanine substitution on the temperature threshold (top graph) and temperature coefficient (bottom graph) measured from the rising phase of whole-cell membrane currents induced by a $25-48^{\circ} \mathrm{C}$ heat ramp $\left(10^{\circ} \mathrm{C} / \mathrm{s}\right)$. The dotted line in the bottom graph indicates the value at which the temperature dependence of the thermally induced currents is considered to be nonspecific [i.e., close to that of the aqueous diffusion limit $(\sim 2)$ ]. $B$, Arrhenius plot of whole-cell currents obtained from representative cells transfected with wild-type (open circles) or I679A-TRPV1 (open squares) normalized at $45^{\circ} \mathrm{C}$ (ordinate, log scale) and plotted against the reciprocal of the absolute temperature (abscissa). The temperature coefficients $Q_{10}$ were determined for each cell over the temperature range at which the Arrhenius plot was linear (dashed lines). C, Activation of channels by depolarizing voltage steps recorded at $25^{\circ} \mathrm{C}$ in extracellular control solution (holding potential, $-70 \mathrm{mV}$; voltage steps from -140 to $+140 \mathrm{mV}$; increment, $+20 \mathrm{mV}$ ). Averaged voltage-current relationships were constructed from responses obtained from three to five independent recordings, such as shown in the inset. They normalized in L669A (open circles), G683A (filled squares), wild-type channel (small filled circles, dotted line). D, Effects of alanine substitution on voltage-dependent gating properties of TRPV1. Voltage-dependent gating parameters, the half-activation voltage $V_{1 / 2}$, and equivalent charge $z$ were estimated from steady-state current-voltage $(I--V)$ relationships obtained at the end of $60 \mathrm{~ms}$ voltage steps from $-140 \mathrm{mV}$ to $+140 \mathrm{mV}$ by fitting them to the following Boltzmann equation: $I=g \times\left(V-E_{\text {rev }}\right) /\left(1+\exp \left(-z F\left(V-V_{1 / 2}\right) / R T\right)\right)$, where $g$ is the whole-cell conductance, $E_{\text {rev }}$ is the reversal potential, and $F, R$, and $T$ have their usual thermodynamic meaning.

TRPV1 channel activation do not need to be strictly coupled (Brauchi et al., 2004).

\section{Discussion}

In this study, we identify the residues within the putative innerpore segment of the vanilloid receptor TRPV1 that when mutated 

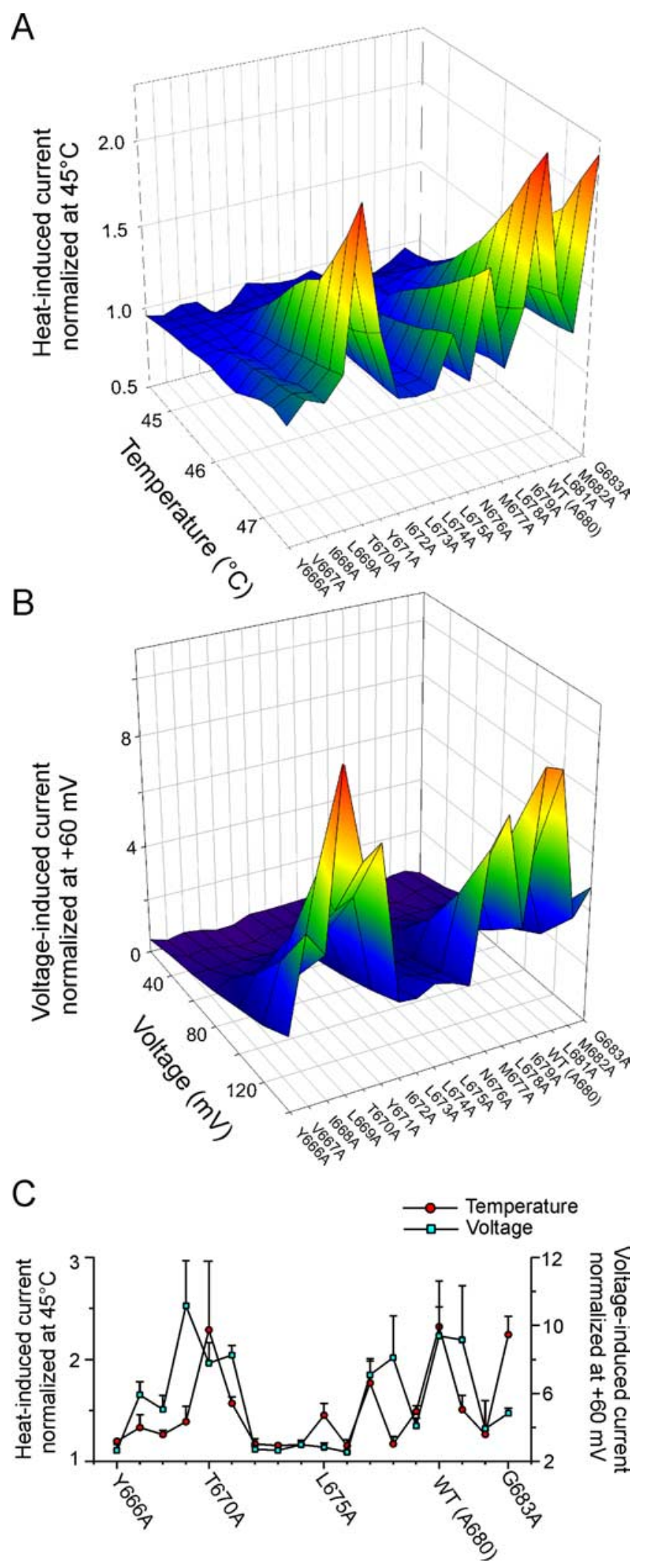

Figure 4. Summary of effects of alanine mutations on heat-induced and voltage-induced activation gating of TRPV1. $\boldsymbol{A}$, Averaged current-temperature plots of normalized heat-evoked responses. $\boldsymbol{B}$, Averaged voltage-current relationships constructed from responses obtained from 3-11 independent recordings, such as in Figure $3 \boldsymbol{C}$, under control conditions at $25^{\circ} \mathrm{C} . \boldsymbol{C}$, Summary of effects of TRPV1 alanine mutations on heat-induced and voltage-induced activation gating. The maximal magnitude of the responses to heat $\left(47.5^{\circ} \mathrm{C}\right.$; left ordinate) compared with the maximal magnitude of the responses to voltage ( $+140 \mathrm{mV}$; right ordinate) is shown. Data are means \pm SEM of 3-11 separate determinations. affect the chemical, thermal, and voltage sensitivity of the channel.

We can be reasonably certain that, in most cases, the reduced magnitudes of the responses to capsaicin or heat are not attributable to reduced expression levels or plasma membrane targeting, because (1) Western blots confirmed that all of the mutants tested were properly routed to the plasma membrane and expressed to a similar extent to that of wild-type TRPV1, (2) simultaneous application of heat and capsaicin revealed considerable differences in the relative cross-sensitization capacity among the mutants, (3) several mutants were less specifically responding to heat (i.e., their current-temperature relationships were qualitatively different from that in the wild-type channel), and, moreover, (4) although there was a substantial overlap between the temperature-dependent and voltage-dependent profiles of the TRPV1 channel, the deviations cannot be overlooked (Fig. 4C). Although changes in channel unitary conductance could also contribute to the observed reduction in capsaicin and heatevoked current amplitude, in most cases, the observed functional changes are likely directly related to the ability of the channel to gate-open in response to an appropriate stimulus. First, the mutations greatly affecting capsaicin activation caused a leftward shift in the temperature threshold for activation; second, except for T670A, L681A, and G683A, the mutations strongly reduced the temperature coefficient in all mutants tested; and third, most of the alanine mutations shifted the channel activation potential toward more positive voltages (Fig. 3).

We found that certain mutations only affect some stimulus modalities but not others. The mutations L669A, Y671A, and M677A elicited large effects on heat-dependent activation but only modest effects on capsaicin- and voltage-dependent activation. These results may indicate that some of the residues differentially affect allosteric coupling between the activation site(s) (capsaicin-binding site, temperature or voltage sensor) and the movement of the gate. In the case of Y671A, this explanation is also supported by the recent finding that this aromatic residue is apparently involved in structural rearrangements of the TRPV1 channel protein leading to capsaicin-induced desensitization (Mohapatra et al., 2003). To explain the diverse effects of mutations on the distinct modalities of the TRPV1 channel protein, it is also important to keep in mind that, if there indeed exists a common gating mechanism, it manifests itself under different physical conditions: once at $47^{\circ} \mathrm{C}$ and $-70 \mathrm{mV}$ and once at $25^{\circ} \mathrm{C}$ and $+140 \mathrm{mV}$.

Site-directed mutagenesis studies have proved to be contributory in obtaining new insights into the mechanism of the gating of several P-loop voltage-gated and ligand-gated ion channels, namely $\mathrm{K}^{+}$(McPhee et al., 1995; Mitcheson et al., 2000; Hackos et al., 2002), $\mathrm{Na}^{+}$(Ragsdale et al., 1994; McPhee et al., 1995; Yarov-Yarovoy et al., 2002; Seebohm et al., 2006), $\mathrm{Ca}^{2+}$ (Raybaud et al., 2007), or cyclic nucleotide-gated (Flynn and Zagotta, 2003) channels. To what extent may our results be correlated with these studies and which of the residues might serve as a molecular hinge in the S6 segment? Given that TRPV1 can be activated by several different stimuli and these have strong synergistic effects, it might be expected that the gating mechanism of this channel is much more complex than of those with only one activation pathway. On the other hand, single-channel kinetic studies on the recombinant TRPV1 channel (Hui et al., 2003; Liu et al., 2003; Ryu et al., 2003) revealed similar kinetic rates involved in the heat-, capsaicin-, and $\mathrm{pH}$-induced activation/deactivation processes, which suggests that a common gating machin- 
ery may be involved in the activation pathways induced by different stimuli.

To explain our results, we used a combination of energetic and homology modeling to construct a three-dimensional molecular model of the fourfold symmetric pore module (S5-P-S6) 4 of the TRPV1 channel (see Materials and Methods). This homology model was based on an identity of $\sim 18 \%$ and a homology of $\sim 43 \%$ between the rat TRPV1 amino acid sequence M572-E692 and the KcsA protein, the structure of which has been solved to a resolution of $1.9 \AA$ (code 1R3J from the Protein Data Bank). At $300 \mathrm{~K}$, the first $20 \mathrm{~ns}$ $\mathrm{MD}$ simulation in a POPC bilayer yielded channel monomers with the inner helices bent; the monomers were not fully symmetrical and exhibited a different secondary structure content. A new tetramer, built from the bended monomer with the highest secondary structure content, was then simulated for an additional $20 \mathrm{~ns}$ and finally led to a channel with all four inner helices symmetrically bent in the region from N676 to A680, splayed at the intracellular ends (Fig. 5). On average, the pore diameter was predicted to be $\sim 6.5 \AA$ in the selectivity filter region and narrowed in the proposed inner pore region to $\sim 3.8 \AA$ at I679. Because of its position in the most constricted region, N676 might be suspected to play an important role in the conformational changes leading to the opening of the channel; however, nothing can be inferred from our data because the alanine mutant (although present at the plasma membrane) failed to express significant whole-cell currents.

The new findings of this study provide essential evidence in favor of a possible functional role for several residues within the inner-pore helix, making them candidates for sites of the gating apparatus of the TRPV1 channel. This portion of the TRPV1 protein does not possess a "classical" point of local flexibility like glycine or proline, thus our model cannot directly lend structural support for identifying a gating hinge. To gain additional information toward a possible structural explanation of our data, we additionally conducted a stability study in vacuo of the TRPV1 S6 monomers gained by homology modeling (Fig. $5 E$ and supplemental Figure S1, available at www.jneurosci.org as supplemental material). Generally, a simulation of the transmembrane section of a protein leads to a quick unfolding or refolding, because the stabilizing lipids are missing. On this "denaturation" pathway, changes should occur first in the most flexible parts of the structure and later in the other parts. Figure $5 E$ shows that after 10 ps simulation, the S6 structure was still a single helix; however, after 70 ps, a bending appeared in the region around T670. A comparison of the average temperature-dependent activation profile for the individual mutants with that obtained for averaged voltagedependent activity (Fig. 4) gives the impression that there are, in fact, two predominant regions within the inner-pore helix: one centered around T670 and the second centered around the native A680, separated by a tract of large aliphatic residues, I672-L675.
These two regions coincide with the two bending regions of the transmembrane helix that were identified and described above by computer modeling techniques. Remarkably, aligning the innerpore region of TRPV 1 with $\mathrm{KcsA}$, Shaker, $\mathrm{Ca}_{\mathrm{v}}$, and $\mathrm{Na}_{\mathrm{v}}$ channels places threonine 670 into the position of the "universal" gating hinge glycine (Fig. 1A). In addition, A680 in wild-type TRPV1 corresponds to the position of the first glycine in the $G(X)_{3} G$ motif, the role of which has been highlighted recently in the gating of high-voltage-activated $\mathrm{Ca}^{2+}$ channels (Raybaud et al., 2007). Interestingly, these voltage-gated ion channels are most closely related to the TRP channel family.

Our results provide functional support for the role of the putative inner-pore region in controlling the gating of the vanilloid receptor TRPV1 channel. Overall, these results confirm that most of the residues within the inner-pore region of the TRPV1 channel are sensitive to alanine substitution with respect to at least one stimulus modality and that most of the mutations lead to a reduction in the degree of specificity to temperature stimuli. Furthermore, we demonstrated the periodicity of the functional effects of the substitutions, which is consistent with an $\alpha$-helical structure of the putative inner-pore region and its active role in gating. Given that a fiveresidue sequence, NMLIA, within the sixth transmembrane domain of the TRPV1 protein is the most strongly conserved across the TRPrelated proteins, the results obtained in this study may also be gen- 
erally important in understanding the processes of gating in other channels.

\section{References}

Berendsen HJC, Postma JPM, van Gunsteren WF, DiNola A, Haak JR (1984) Molecular-dynamics with coupling to an external bath. J Chem Phys 81:3684-3690.

Brauchi S, Orio P, Latorre R (2004) Clues to understanding cold sensation: thermodynamics and electrophysiological analysis of the cold receptor TRPM8. Proc Natl Acad Sci USA 101:15494-15499.

Caterina MJ, Schumacher MA, Tominaga M, Rosen TA, Levine JD, Julius D (1997) The capsaicin receptor: a heat-activated ion channel in the pain pathway. Nature 389:816-824.

Dittert I, Benedikt J, Vyklicky L, Zimmermann K, Reeh PW, Vlachova V (2006) Improved superfusion technique for rapid cooling or heating of cultured cells under patch-clamp conditions. J Neurosci Methods 151:178-185.

Dodier Y, Banderali U, Klein H, Topalak O, Dafi O, Simoes M, Bernatchez G, Sauve R, Parent L (2004) Outer pore topology of the ECaC-TRPV5 channel by cysteine scan mutagenesis. J Biol Chem 279:6853-6862.

Doyle DA, Morais Cabral J, Pfuetzner RA, Kuo A, Gulbis JM, Cohen SL, Chait BT, MacKinnon R (1998) The structure of the potassium channel: molecular basis of $\mathrm{K}+$ conduction and selectivity. Science 280:69-77.

Ferrer-Montiel A, Garcia-Martinez C, Morenilla-Palao C, Garcia-Sanz N, Fernandez-Carvajal A, Fernandez-Ballester G, Planells-Cases R (2004) Molecular architecture of the vanilloid receptor. Insights for drug design. Eur J Biochem 271:1820-1826.

Flynn GE, Zagotta WN (2003) A cysteine scan of the inner vestibule of cyclic nucleotide-gated channels reveals architecture and rearrangement of the pore. J Gen Physiol 121:563-582.

Gunthorpe MJ, Smith GD, Davis JB, Randall AD (2001) Characterisation of a human acid-sensing ion channel (hASICla) endogenously expressed in HEK293 cells. Pflügers Arch 442:668-674.

Hackos DH, Chang TH, Swartz KJ (2002) Scanning the intracellular S6 activation gate in the shaker K+ channel. J Gen Physiol 119:521-532.

Hui K, Liu B, Qin F (2003) Capsaicin activation of the pain receptor, VR1: multiple open states from both partial and full binding. Biophys $\mathrm{J}$ 84:2957-2968.

Jiang Y, Lee A, Chen J, Cadene M, Chait BT, MacKinnon R (2002) Crystal structure and mechanism of a calcium-gated potassium channel. Nature 417:515-522.

Kuzhikandathil EV, Wang H, Szabo T, Morozova N, Blumberg PM, Oxford GS (2001) Functional analysis of capsaicin receptor (vanilloid receptor subtype 1) multimerization and agonist responsiveness using a dominant negative mutation. J Neurosci 21:8697-8706.

Laskowski RA, MacArthur MW, Moss DS, Thornton JM (1993) PROCHECK: a program to check the stereochemical quality of protein structures. J Appl Crystallogr 26:283-291.

Liu B, Hui K, Qin F (2003) Thermodynamics of heat activation of single capsaicin ion channels VR1. Biophys J 85:2988-3006.

McPhee JC, Ragsdale DS, Scheuer T, Catterall WA (1995) A critical role for transmembrane segment IVS6 of the sodium channel alpha subunit in fast inactivation. J Biol Chem 270:12025-12034.

Mitcheson JS, Chen J, Lin M, Culberson C, Sanguinetti MC (2000) A structural basis for drug-induced long QT syndrome. Proc Natl Acad Sci USA 97:12329-12333.

Mohapatra DP, Wang SY, Wang GK, Nau C (2003) A tyrosine residue in
TM6 of the vanilloid receptor TRPV1 involved in desensitization and calcium permeability of capsaicin-activated currents. Mol Cell Neurosci 23:314-324.

Nilius B, Prenen J, Janssens A, Owsianik G, Wang C, Zhu MX, Voets T (2005a) The selectivity filter of the cation channel TRPM4. J Biol Chem 280:22899-22906

Nilius B, Talavera K, Owsianik G, Prenen J, Droogmans G, Voets T (2005b) Gating of TRP channels: a voltage connection? J Physiol (Lond) 567:35-44.

Owsianik G, Talavera K, Voets T, Nilius B (2006) Permeation and selectivity of TRP channels. Annu Rev Physiol 68:685-717.

Planells-Cases R, Garcia-Sanz N, Morenilla-Palao C, Ferrer-Montiel A (2005) Functional aspects and mechanisms of TRPV1 involvement in neurogenic inflammation that leads to thermal hyperalgesia. Pflügers Arch 451:151-159.

Ragsdale DS, McPhee JC, Scheuer T, Catterall WA (1994) Molecular determinants of state-dependent block of $\mathrm{Na}+$ channels by local anesthetics. Science 265:1724-1728.

Raybaud A, Dodier Y, Bissonnette P, Simoes M, Bichet DG, Sauve R, Parent L (2006) The role of the $\mathrm{G}(\mathrm{X}) 9 \mathrm{G}(\mathrm{X}) 3 \mathrm{G}$ motif in the gating of high-voltage activated Ca2+ channel. J Biol Chem 281:39424-39436.

Ryu S, Liu B, Qin F (2003) Low pH potentiates both capsaicin binding and channel gating of VR1 receptors. J Gen Physiol 122:45-61.

Sali A, Blundell TL (1993) Comparative protein modelling by satisfaction of spatial restraints. J Mol Biol 234:779-815.

Seebohm G, Strutz-Seebohm N, Ureche ON, Baltaev R, Lampert A, Kornichuk G, Kamiya K, Wuttke TV, Lerche H, Sanguinetti MC, Lang F (2006) Differential roles of $\mathrm{S} 6$ domain hinges in the gating of KCNQ potassium channels. Biophys J 90:2235-2244.

Szallasi A, Cruz F, Geppetti P (2006) TRPV1: a therapeutic target for novel analgesic drugs? Trends Mol Med 12:545-554.

Tominaga M, Tominaga T (2005) Structure and function of TRPV1. Pflügers Arch 451:143-150.

Tominaga M, Caterina MJ, Malmberg AB, Rosen TA, Gilbert H, Skinner K, Raumann BE, Basbaum AI, Julius D (1998) The cloned capsaicin receptor integrates multiple pain-producing stimuli. Neuron 21:531-543.

Van Der Spoel D, Lindahl E, Hess B, Groenhof G, Mark AE, Berendsen HJ (2005) GROMACS: fast, flexible, and free. J Comput Chem 26: 1701-1718.

Vlachova V, Lyfenko A, Orkand RK, Vyklicky L (2001) The effects of capsaicin and acidity on currents generated by noxious heat in cultured neonatal rat DRG neurones. J Physiol (Lond) 533 3:717-728.

Vlachova V, Teisinger J, Susankova K, Lyfenko A, Ettrich R, Vyklicky L (2003) Functional role of C-terminal cytoplasmic tail of rat vanilloid receptor 1. J Neurosci 23:1340-1350.

Voets T, Janssens A, Droogmans G, Nilius B (2004a) Outer pore architecture of a Ca2+-selective TRP channel. J Biol Chem 279:15223-15230.

Voets T, Droogmans G, Wissenbach U, Janssens A, Flockerzi V, Nilius B (2004b) The principle of temperature-dependent gating in cold- and heat-sensitive TRP channels. Nature 430:748-754.

Yarov-Yarovoy V, McPhee JC, Idsvoog D, Pate C, Scheuer T, Catterall WA (2002) Role of amino acid residues in transmembrane segments IS6 and IIS6 of the Na + channel alpha subunit in voltage-dependent gating and drug block. J Biol Chem 277:35393-35401.

Yeh BI, Kim YK, Jabbar W, Huang CL (2005) Conformational changes of pore helix coupled to gating of TRPV5 by protons. EMBO J 24:32243234 . 